\title{
Changes in foot pressure on the ground during mae-geri kekomi (front kick) in karate athlete - case study
}

\author{
Cynarski Wojciech J. ${ }^{1 \mathrm{ABCDE}}$, Wąsik Jacek ${ }^{* 2 \mathrm{ABCDE}}$, Szymczyk Daniel ${ }^{3 \mathrm{BDE}}$, Vences de Brito Antonio ${ }^{4,5 D E}$
}

${ }^{1}$ Faculty of Physical Education, University of Rzeszow, Rzeszow, Poland

${ }^{2}$ Institute of Physical Education, Tourism and Physiotherapy, Jan Dlugosz University in Czestochowa, Poland

${ }^{3}$ Institute of Physiotherapy, Faculty of Medicine, University of Rzeszow, Rzeszow, Poland

${ }^{4}$ Sports Sciences School of Rio Maior - Polytechnic Institute of Santarém, Portugal

${ }^{5}$ Investigation Center in Life Quality (CIEQV), Portugal

Authors' Contribution: A - Study design; B - Data collection; C - Statistical analysis; D - Manuscript Preparation; E - Funds Collection.

\begin{abstract}
Purpose:

Biomechanical analysis of martial art techniques are objective and quantitative method, which may be useful in training practice for the purpose of improving these techniques as well as monitoring and enhancing athletes performance. The aim of this study was to assess selected kinetic parameters and lower limb loading during karate front kick, using force platforms, for both kicking and support leg.

Material: $\quad$ Karate athlete, second dan, black belt holder in Idokan style, participated in this case study. The subject performed front kick in the air (without a physical target), both for his right and left leg, while standing on the force platforms. For the purpose of kinetic data recording two force platforms were used in this study (Kistler force plate, type 9286AA, Kistler, Switzerland). Selected kinetic parameters for kicking and support leg as well as front kick execution time were analysed.

Results: $\quad$ Results of our case study revealed higher values of the kinetic parameters for the right leg, comparing to the left leg. This results confirm athlete's declaration of being right leg dominant.

Conclusions: $\quad$ Analysis of selected kinetic parameters may be useful in identifying lower leg laterization (leg dominance), as well as existing asymmetries between the dominant and non-dominant leg in karate and other martial art athletes.

Keywords: martial art, karate, kinetics, frontal kick, force platform
\end{abstract}

\section{Introduction}

Biomechanics of martial arts allows for identifying and better understanding the factors that can influence a successful performance at a competition, show or during a fight. This knowledge can also enhance the ability of proper learning and teaching of the karate strokes and techniques. The frontal kick is relatively easy to learn, but it is difficult to master karate technique. It includes rotational movements performed to produce high-speed movement of the lower leg kinematic chain segments. In the available scientific literature concerning the biomechanics of combat sports and martial arts it is not one of the most commonly analysed kicks, while the most attention is devoted to the roundhouse kick [1-4].

Previous studies concerning biomechanical analysis of this type of strokes have already provided some basic information. The average kicking time is about 0.63 seconds and the average speed is $10.40 \mathrm{~m} / \mathrm{s}$ for the taekwon-do athletes [5]. It was found that the speed of the front kick technique mostly depended on the movement velocity in the knee joint and kicking time (duration of the kick) [5, 6]. Sorensen et al. [7] reported that in the case of high front kick, the thigh slowing is due to movement which depends on the initial movement in the lower limb rather than the inhibition activity. Differences in kinematics during performance of the frontal kick were observed in Shotokan karate athletes [8]. Depending on the athletes level of experience, more repeatability in the movement kinematics were observed, especially in the

(C) Cynarski Wojciech J., Wąsik Jacek, Szymczyk Daniel,

Vences de Brito Antonio, 2018

doi:10.15561/20755279.2018.0102 pre-loading phase, which precedes the attack phase. It was found that the duration of the kick and the repeatability of the lower limb movement could be useful when selecting the top karate players and monitoring their preparation status. It is recommended that in karate sports training this type of kicking should be performed using physical targets [9].

We know that distance from the physical target affects kinetics of the kick [10]. Increasing distances reduces the impact force and increases the reaction time.

Speed is an important element of success in combat sports and self defence. Sometimes, the victory or the defeat in the confrontation depends on the athletes speed of reaction. However, it has been suggested that precision and kicking speed are opposite priorities [11,12].

Cynarski et al. [13] described that the karate front kick (mae-geri kekomi) should be done very precisely. All of the aforementioned movements should form a single fluent motion without any pauses, so that the first movement of raising the knee adds impact to the violent kick. If the kick is towards the chudan zone, i.e. at the height of the solar plexus, the striking surface (chusoku) shall move towards the target along a straight line in the final section of motion. The front kick is best performed from a stable stance. It can be done with the front leg or the back leg, as well as when stepping forward.

Therefore, the purpose of this study was to assess selected kinetic parameters and lower limb loading during karate front kick, using force platforms. Changes of the force of pressure applied to the ground for both kicking and support leg were recorded and analysed. 
We hypothesised that we can observe some difference between the right and left leg due to the lateralization (lower leg dominance).

\section{Material and methods}

\section{Participants}

Karate athlete, second dan black belt holder in Idokan style [14], participated in this case study (age: 36 years; body mass: $97 \mathrm{~kg}$; height: $177 \mathrm{~cm}$ ).

The study procedure was approved by the Ethical Committee of the University of Rzeszow as a meeting the criteria of Ethical Conduct for Research Involving Humans. A subject participating in this study was informed about all testing procedures and has signed written informed consent.

Procedure

The athlete participating in our study was asked to perform front kick in the air (without a physical target), from zenkutsu-dachi standing position, both for his right and left leg, while standing on the force platforms. For the purpose of kinetic data recording two force platforms were used in this study (Kistler force plate, type 9286AA, Kistler, Switzerland), in order to acquire kinetic data for both kicking and support leg. Changes in pressure force applied to the ground for both legs were recorded and analysed. Following parameters for the support leg were calculated: $\mathrm{P}_{\mathrm{N}}$ - maximum force applied to the ground (pre-loading phase) $\mathrm{P}_{\mathrm{K}}$ - maximum force applied to the ground during leg extension phase of the kicking leg (attack phase for the kicking leg). For the kicking leg following parameters were determined: FT - maximum force applied to the ground (pre-loading phase), FE maximum force applied to the ground after the kick execution, $\mathrm{T}$ - front kick execution time (kicking foot with no contact with the ground)

\section{Statistical analysis}

Due to the fact that our research was a case study, there was no statistical analysis of the results applied [15]. Data in the results section of this article is presented as an average (maximum) value of selected kinetic parameters obtained from the force platform, as well as in the graphs.

\section{Results}

Table 1 presents the value of selected kinetic parameters of the right leg front kick performed by the karate player as well as the right leg front kick execution time, which is 0,95 second. Figure 1 is the graphical presentation of the kinetic parameters, acquired using the force platforms, both for kick leg (right) and support leg (left).

Table 2 presents the value of selected kinetic

Table 1. Selected kinetic parameters for right leg front kick

\begin{tabular}{llllll}
\hline & $\mathbf{P}_{\mathbf{N}}[\mathbf{N}]$ & $\mathbf{F}_{\mathbf{T}}[\mathbf{N}]$ & $\mathbf{T}[\mathbf{s}]$ & $\mathbf{F}_{\mathbf{E}}[\mathbf{N}]$ & $\mathbf{P}_{\mathbf{K}}[\mathbf{N}]$ \\
\hline Kick leg (right) & - & 1013,049 & 0,95 & 1208,694 & - \\
Support leg (left) & 1295,668 & - & - & - & 1025,557 \\
\hline
\end{tabular}

Parameters for the kick leg (right) - $\mathrm{F}_{\mathrm{T}}$ - maximum force applied to the ground (pre-loading phase), $\mathrm{F}_{\mathrm{E}}-$ maximum force applied to the ground after the kick execution, $T$ - front kick execution time (kicking foot with no contact with the ground)

Parameters for the support leg (left) - $P_{N}$ - maximum force applied to the ground (pre-loading phase) $P_{K}-$ maximum force applied to the ground during leg extension phase of the kicking leg (attack phase of the kicking leg)

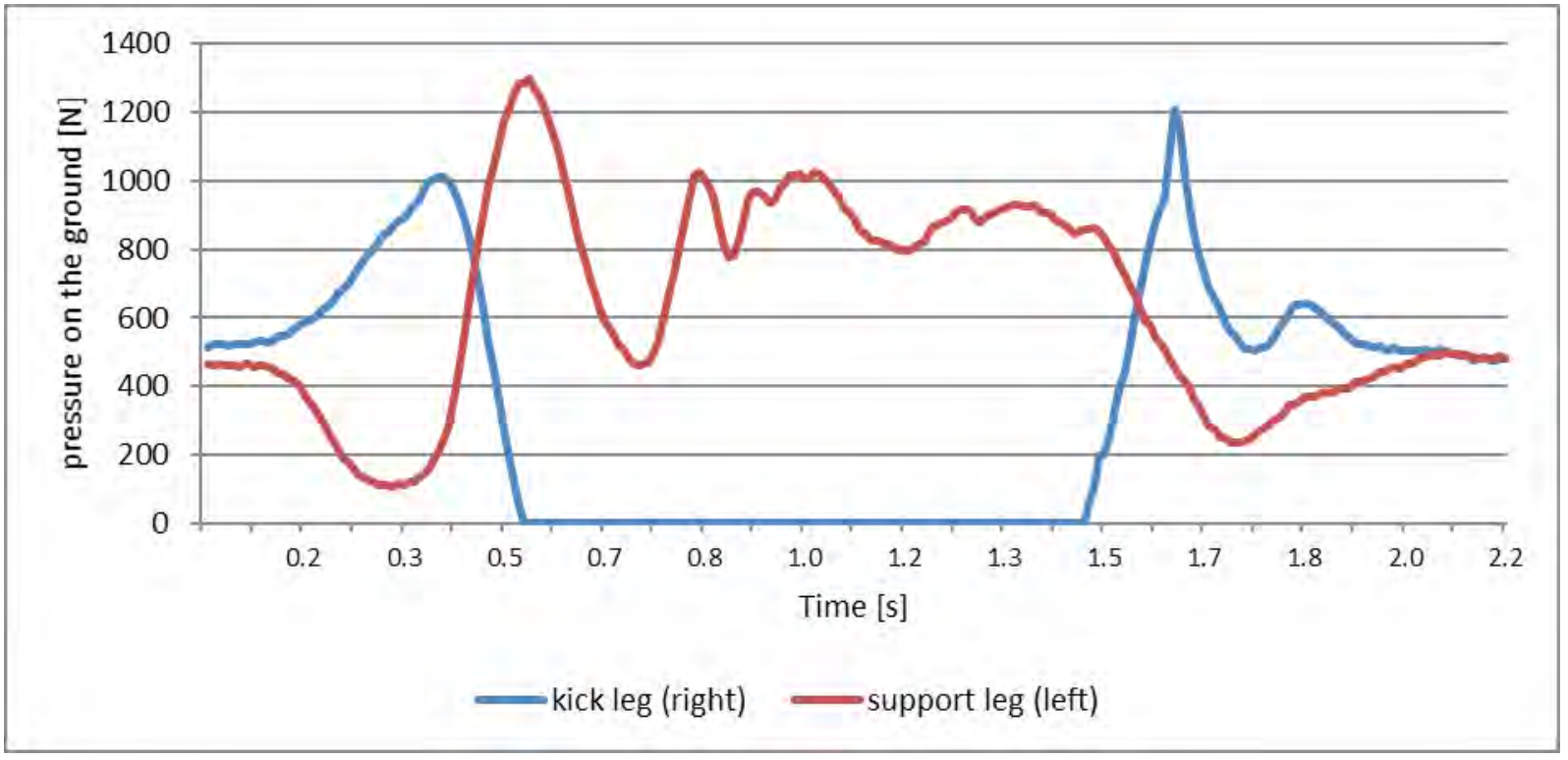

Fig. 1. Changes of foot pressure on the ground while performing the right leg front kick 
parameters of the left leg front kick, as well as the left leg front kick execution time, which is 0,89 second. Figure 2 is the graphical presentation of the kinetic parameters, acquired using the force platforms, both for the kick leg (left) and support leg (right).

\section{Discussion}

Our case study results revealed that the maximum load on the support leg while performing a karate front kick was approximately 1295-1462 N - for the left and right leg respectively (tables 1,2). It has been reported that in taekwon-do players performing the jump front kick, the values of ground reaction forces were about $400 \mathrm{~N}$ higher, comparing to our results [16]. During the kicking leg extension phase (in the knee joint), the maximum values of the ground reaction forces for the support leg are around 1024-1025 $\mathrm{N}$ - for the left and right leg respectively (tables 1,2). With a constant body weight, the high speed of the kicking leg is associated with a large change in momentum. A large momentum (change in momentum) informs us about a great force impulse [17]. Thus, according to the third principle of dynamics, this should be reflected in the force of pressure on the ground, recorded using force platforms. In the future, it is worth checking if there is a correlation between the speed of the kicking leg and the ground reaction forces of the support leg.

In our study the front kick execution time (time when the kicking leg has no contact with the ground) was 0,89 seconds for the left leg and 0, 95 seconds for the right leg. Within this time, the kicking leg was extended and then returned to the starting position.

The athlete participated in this study declared that his dominant leg was the right leg. Results of our study confirmed this and revealed higher values of selected kinetic parameters for the right leg, comparing to the left leg. The maximum value of the force applied to the ground in the pre-loading phase - $\mathrm{F}_{\mathrm{T}}$ (before the attackphase) was almost $63 \mathrm{~N}$ higher for the right leg comparing to the left leg (tables 1,2). Interestingly, similar results were observed concerning maximum force applied to the ground with kicking leg after the kick execution $\left(\mathrm{F}_{\mathrm{E}}\right)$ $1082 \mathrm{~N}$ for the left leg and $1208 \mathrm{~N}$ for the right leg, with a difference of $126 \mathrm{~N}$ between the right and left leg (tables 1,2).

Such a methods of biomechanical analysis of karate

Table 2. Selected kinetic parameters for left leg front kick

\begin{tabular}{llllll}
\hline & $\mathbf{P}_{\mathbf{N}}[\mathbf{N}]$ & $\mathbf{F}_{\boldsymbol{T}}[\mathbf{N}]$ & $\mathbf{T}[\mathbf{s}]$ & $\mathbf{F}_{\mathbf{E}}[\mathbf{N}]$ & $\mathbf{P}_{\boldsymbol{K}}[\mathbf{N}]$ \\
\hline Kick leg (left) & - & 950,516 & 0,892 & 1082,424 & - \\
Support leg (right) & 1462,067 & - & - & - & 1024,301 \\
\hline
\end{tabular}

Parameters for the kick leg (left) - $F_{T}$ - maximum force applied to the ground (pre-loading phase), $F_{E}$ - maximum force applied to the ground after the kick execution, $T$ - front kick execution time (kicking foot with no contact with the ground)

Parameters for the support leg (right) - $P_{N}$ - maximum force applied to the ground (pre-loading phase) $P_{K}-$ maximum force applied to the ground during leg extension phase of the kicking leg (attack phase of the kicking leg)

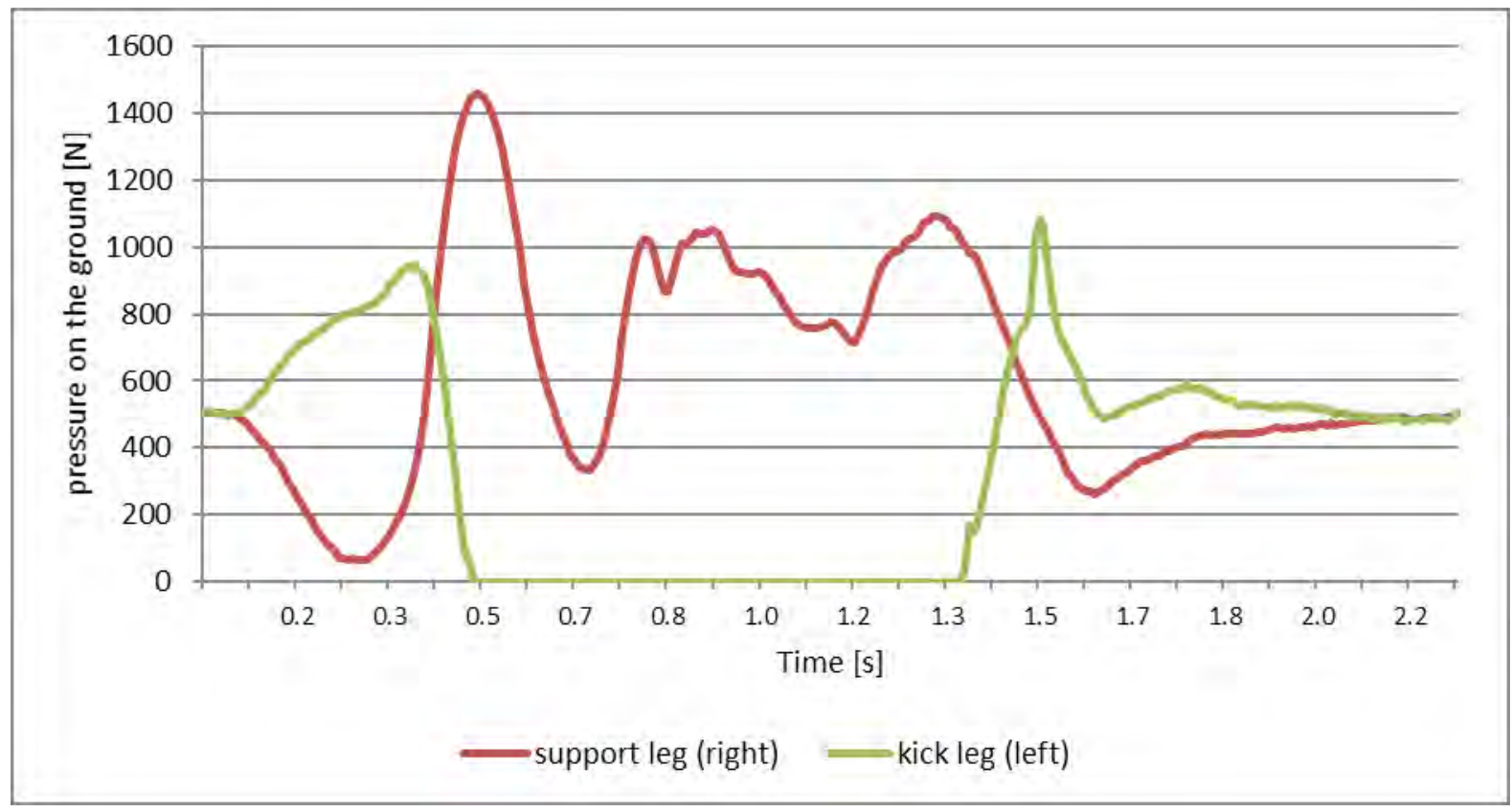

Fig. 2. Changes of foot pressure on the ground while performing the left leg front kick 
techniques (as well as other martial arts and combat sports) provides precise and quantitative information about the movement, which may enable to improve these techniques in training practice and enhance athletes' performance $[13,18]$.

Our study has some limitation, due to its single-subject case study character. Although the results are promising and they can indicate the need for its continuation on a bigger group of subjects, which enables for its appropriate statistical analysis. The results presented here may also provide some initial, basic data for further comparisons in a similar research. They can also indicate the need for interdisciplinary exploration and research, which includes the area of therapeutic and rehabilitation activities inspired by martial arts [19-23].

\section{Conclusions}

Due to the study limitations, it is impossible to present any direct conclusion or statement. We can assume that analysis of selected kinetic parameters may be useful in identifying lower leg laterization (leg preference), as well as existing asymmetries between the dominant and non-dominant leg in karate and other martial art athletes. Besides the results of our study can highlight the importance of quantitative, biomechanical analysis of martial art techniques for the purpose of their improving in training and enhancing athletes performance.

\section{Conflict of interests}

The authors declare that there is no conflict of interests.

\section{References}

1. Serina ER, Lieu DK. Thoracic injury potential of basic competition taekwondo kicks. J Biomech. 1991;24(10):951960.

2. Wąsik J. The structure of the roundhouse kick on the example of a European Champion of taekwon-do. Archives of Budo. 2010;6(4):211-6

3. Kalichová M, Vít M, Reguli Z, Pieter W. Kinematic characterization of the Capoeira Bencao Kick. Ido Movement for Culture. Journal of Martial Arts Anthropology. 2012;12(4):7-11.

4. Gavagan CJ, Sayers MGL. A biomechanical analysis of the roundhouse kicking technique of expert practitioners: A comparison between the martial arts disciplines of Muay Thai, Karate, and Taekwondo. PLoS ONE. 2017;12(8): e0182645. doi:10.1371/journal.pone.0182645

5. Wąsik J, Czarny W, Małolepszy E, Małolepsza T. Kinematics of taekwon-do front kick. Arch Budo Sci Martial Art Extreme Sport. 2015;11:23-9.

6. Vences Brito AM, Colaço Branco MA, Cordeiro Fernandes RM, Rodrigues Ferreira MA, Fernandes Orlando JSM, Figueiredo Abel A, et al. Avaliação eletromiográfica e cinemática do pontapé Mae-geri em karatecas de elite competitiva e karatecas cinto negro não competidores. Revista UIIPS. 2014;3(2):60-79.

7. Sorensen H, Zacho M, Simonsen EB, Dyhre-Poulsen P, Klausen K. Dynamics of themartial artshigh frontkick. JSports Sci. 1996;14(6):483-95. doi:10.1080/02640419608727735

8. Pozo J, Bastien G, Dierick F. Execution time, kinetics, and kinematics of the mae-geri kick: comparison of national and international standard karate athletes. J Sports Sci. 2011;29(14):1553-61. doi:10.1080/02640414.2011.605164

9. Portel BS, Barbosa MR, Cavazzotto TG, Tartaruga MP. Kinematics analysis of the front kick with and without impact on traditional karate. Arch Budo Sci Martial Art Extreme Sport. 2014;10:47-51.

10.Falco C, Alvarez O, Castillo I, Estevan I, Martos J, Mugarra $\mathrm{F}$, et al. Influence of the distance in a roundhouse kick's execution time and impact force in Taekwondo. $J$ Biomech. 2009; 42(3):242-8. doi: 10.1016/j.jbiomech.2008.10.041.

11.Wąsik J, Shan G. Target Effect on the Kinematics of Taekwondo Roundhouse Kick. Is the Presence of a Physical Target a Stimulus, Influencing Muscle-power Generation? Acta of Bioengineering and Biomechanics. 2015; 17(4):115120.

12.Wąsik J, Góra T. Impact of target selection on front kick kinematics in taekwondo - pilot study. Physical Activity
Review. 2016;4:57-61. doi: 10.16926/par.2016.04.07

13.Cynarski WJ, Wajs W, VencesBrito A. Improving the movements of basic karate techniques with the use of motion capture and mathematical modeling. The concept of a research project. Ido Movement for Culture. Journal of Martial Arts Anthropology. 2014;14(1):39-53.

14.Cynarski WJ, Sieber L. 40 Years of Zendo Karate Tao-Te-Tao and Idokan Karate (1975-2015). Ido Movement for Culture. Journal of Martial Arts Anthropology. 2016;16(1):11-17; doi: 10.14589/ido.16.1.2

15.Skinner J., Edwards A., Corbett B. Research methods for sport management, Routledge: London - New York; 2015.

16. Wąsik J. The structure and influence of different flying high front kick techniques on the achieved height on the example of taekwon-do athletes. Archives of Budo. 2012;8:45-50.

17.Hay J.G. (1993), The biomechanics of sport techniques. $4^{\text {th }}$ edition. Prentice Hall: Englewood Cliffs NJ; 1993.

18.VencesBrito AM, Colaço Branco MA, Cordeiro Fernandes RM, Rodrigues Ferreira MA, Fernandes Orlando JSM, Abreu Figueiredo AA et al. Characterization of kinesiological patterns of the frontal kick, maegeri, in karate experts and nonkarate practitioners. Revista de Artes Marciales Asiáticas. 2014;9(1):20-31.

19.Sogabe A. Influence of difference in knee alignment on site of pain and psychological state after long-distance walking. Physical Activity Review. 2013;1:1-9.

20.Ortenburger D, Wąsik J, Góra T. Report of the 1st World Congress on Health and Martial Arts in Interdisciplinary Approach, 17-19 September 2015, Czestochowa. Physical Activity Review. 2015;3:49-51.

21. Ortenburger D, Wąsik J, Bukova A, Góra T. Comparison of strategies used by patients undergoing treatment for chronic pain people performing taekwon-do - a pilot study. Ido Movement for Culture. Journal of Martial Arts Anthropology. 2016;16(3):40-46. doi:10.14589/ido.16.3.5

22.Pobratyn A, Ciesielska Z, Lesiak K, Ziental A. Eastern Martial Arts in cinematography and popularising a healthy lifestyle - interpretation sketch. Physical Activity Review. 2017;5:78-82. doi:10.16926/par.2017.05.11

23.Szerla M, Ortenburger D, Kluszczyński M, Wyszomierska J. Exercise and psychological factors in low back pain. Physical Activity Review. 2017;5:6-9. doi:10.16926/par.2017.05.02 
Information about the authors:

Cynarski W.; http://orcid.org/0000-0003-1252-5456; ela_cyn@wp.pl; University of Rzeszów; ul.Towarnickiego 3, Rzeszów, 35-959, Poland.

Wąsik J. (Corresponding author); http://orcid.org/0000-0002-6285-7283; jwasik@konto.pl; Jan Dlugosz University in Czestochowa; ul. Waszyngtona 4/8, Czestochowa, 42-200, Poland.

Szymczyk D.; http://orcid.org/0000-0002-0455-3759; dszymczyk@ur.edu.pl; Institute of Physiotherapy, University of Rzeszow; Ul. Warszawska 26 a, Rzeszów, 35-205, Poland..

Vences de Brito A.; http://orcid.org/0000-0001-6964-8559; abrito@esdrm.ipsantarem.pt; Sports Sciences School of Rio Maior PISantarém; Av. Dr. Mário Soares, n¹10 2040 - 413 Rio Maior, Portugal.

Cite this article as: Cynarski Wojciech J, Wasik Jacek, Szymczyk Daniel, Vences de Brito Antonio. Changes in foot pressure on the ground during mae-geri kekomi (front kick) in karate athlete - case study. Physical education of students, 2018;22(1):12-16. doi:10.15561/20755279.2018.0102

The electronic version of this article is the complete one and can be found online at: http://www.sportedu.org.ua/index.php/PES/issue/archive

This is an Open Access article distributed under the terms of the Creative Commons Attribution License, which permits unrestricted use, distribution, and reproduction in any medium, provided the original work is properly cited (http://creativecommons.org/licenses/by/4.0/deed.en).

Received: 03.11.2017

Accepted: 01.12.2017; Published: 27.02.2018 